The Journal of Nonlinear $\mathbf{S}_{\text {cience and Aplications }}$ http://www.tjnsa.com

\title{
A NOTE ON NEW ESTIMATES FOR DISTANCES IN ANALYTIC FUNCTION SPACES
}

\author{
ROMI SHAMOYAN ${ }^{1}$ AND HAIYING LI ${ }^{2 *}$
}

\begin{abstract}
We provide new estimates for distances from fixed analytic functions to their subspaces in the unit disk. We will enlarge the list of previously known assertions of this type obtained recently by R. Zhao and W. Xu.
\end{abstract}

\section{INTRODUCTION AND PRELIMINARIES}

Let $\mathbb{D}$ be, as usual, the unit disk on the complex plane, $d A(z)$ the normalized Lebesgue measure on $\mathbb{D}$ so that $A(\mathbb{D})=1$ and $d \xi$ be the Lebesgue measure on the circle $\mathrm{T}=\{\xi:|\xi|=1\}$. Let further $H(\mathbb{D})$ be the space of all holomorphic functions on the unit disk $\mathbb{D}$.

For $f \in H(\mathbb{D})$ and $f(z)=\sum_{k} a_{k} z^{k}$, we define the fractional derivative of the function $f$ as usual in the following manner

$$
D^{\alpha} f(z)=\sum_{k=0}^{\infty}(k+1)^{\alpha} a_{k} z^{k}, \alpha \in \mathbb{R} .
$$

We will write $D f(z)$ if $\alpha=1$. Obviously, for all $\alpha \in \mathbb{R}, D^{\alpha} f \in H(\mathbb{D})$.

For $a \in \mathbb{D}$, let $g(z, a)=\log \frac{1}{\left|\phi_{a}(z)\right|}$ be the Green's function for $\mathbb{D}$ with pole at $a$, where $\phi_{a}(z)=\frac{a-z}{1-\bar{a} z}$. For $0<p<\infty,-2<q<\infty,-2<s<\infty,-1<q+s<$ $\infty$, we say that $f \in F(p, q, s)$, if $f \in H(\mathbb{D})$ and

$$
\|f\|_{F(p, q, s)}^{p}=\sup _{a \in \mathbb{D}} \int_{\mathbb{D}}|D f(z)|^{p}\left(1-|z|^{2}\right)^{q} g^{s}(z, a) d A(z)<\infty .
$$

Date: Received: 22 Sep. 2009; Revised: 11 Dec. 2009

* Corresponding author.

2000 Mathematics Subject Classification. Primary 30D45.

Key words and phrases. holomorphic function; distance function; Bloch-type space; Bergman-type classes; unit disk. 
As we know [10], if $0<p<\infty,-2<q<\infty,-2<s<\infty,-1<q+s<$ $\infty, f \in F(p, q, s)$ if and only if

$$
\sup _{a \in \mathbb{D}} \int_{\mathbb{D}}|D f(z)|^{p}\left(1-|z|^{2}\right)^{q}\left(1-\left|\phi_{a}(z)\right|\right)^{s} d A(z)<\infty .
$$

It is known also that $F(2,1,1)=B M O A$.

We recall that weighted Bloch class $\mathbf{B}^{\alpha}(\mathbb{D}), \alpha>0$, is the collection of the analytic functions on the unit disk satisfying

$$
\|f\|_{\mathbf{B}^{\alpha}}=\sup _{z \in \mathbb{D}}|D f(z)|\left(1-|z|^{2}\right)^{\alpha}<\infty .
$$

$\mathbf{B}^{\alpha}(\mathbb{D})$ is a Banach space with the norm $\|f\|_{\mathbf{B}^{\alpha}}$. Note $\mathbf{B}^{1}(\mathbb{D})=\mathbf{B}(\mathbb{D})$ is a classical Bloch class(see [2] and the references there).

The well-known so called "duality" approach to extremal problems in theory of analytic functions leads to the following general formula

$$
\operatorname{dist}_{Y}(g, X)=\sup _{l \in X^{\perp},\|l\| \leq 1}|l(g)|=\inf _{\varphi \in X}\|g-\varphi\|_{Y},
$$

where $g \in Y, X$ is subspace of a normed space $Y, Y \in H(\mathbb{D})$ and $X^{\perp}$ it is orthogonal complement in $Y^{*}$, the dual space of $Y$ and $l$ is a linear functional on $Y$ (see [6]).

Various extremal problems in $H^{p}$ Hardy classes in $\mathbb{D}$ based on duality approach we mentioned were discussed in [3]. In particular for a function $K \in L^{q}(\mathrm{~T})$ the following equality holds (see [3]), $1 \leq p<\infty, \frac{1}{p}+\frac{1}{q}=1$,

$$
\operatorname{dist}_{L^{q}}\left(K, H^{q}\right)=\inf _{g \in H^{q}, K \in L^{q}}\|K-g\|_{H^{q}}=\sup _{f \in H^{p},\|f\|_{H^{p} \leq 1}} \frac{1}{2 \pi}\left|\int_{|\xi|=1} f(\xi) K(\xi) d \xi\right| .
$$

It is well known if $p>1$ then inf-dual extremal problem in analytic $H^{p}$ Hardy classes has a solution, it is unique if an extremal function exists (see [3]).

Note also that extremal problems for $H^{p}$ spaces in multiply connected domains were studied before in [1] and [7].

Various new results on extremal problems in $A^{p}$ Bergman class and it is subspaces were obtained recently by many authors (see [5] and the references there).

In this paper, we will provide direct proofs for estimation of

$$
\operatorname{dist}_{Y}(f, X)=\inf _{g \in X}\|f-g\|_{Y}, X \subset Y, f \in Y, X, Y \subset H(\mathbb{D})
$$

in the unit disk.

Let further $\Omega_{\alpha, \varepsilon}^{k}=\left\{z \in \mathbb{D}:\left|D^{k} f(z)\right|\left(1-|z|^{2}\right)^{\alpha} \geq \varepsilon\right\}, \Omega_{\alpha, \varepsilon}^{0}=\Omega_{\alpha, \varepsilon}$.

Applying famous Fefferman duality theorem, P. Jones proved the following.

Theorem A. ([4], [10]) Let $f \in \mathbf{B}$. Then the following are equivalent:

(a) $d_{1}=\operatorname{dist}_{\mathbf{B}}(f, B M O A)$;

(b) $d_{2}=\inf \left\{\varepsilon: \chi_{\Omega_{1, \varepsilon}^{1}}(z) \frac{d A(z)}{1-|z|^{2}}\right.$ is a Carleson measure $\}$,

where $\chi$ denotes characteristic function of the mentioned set.

Recently, R. Zhao (see [10]) and W. Xu (see [9]) obtained results on distances from Bloch functions to some Möbius invariant function spaces in a relatively 
direct way. The goal of this paper is to develop further their ideas and present new theorems in the unit disk.

In next sections various assertions for distance function will be given. We will indicate proofs of some assertions in details, short sketches in some cases will be also provided.

Throughout the paper, we write $C$ ( sometimes with indexes) to denote a positive constant which might be different at each occurrence (even in a chain of inequalities) but is independent of the functions or variables being discussed.

We will write for two expressions $A \lesssim B$ if there is a positive constant $C$ such that $A<C B$.

\section{Estimates for Dist FUnCtion IN $\mathbb{D}$}

First we define a set as following.

Definition 2.1.

$$
\begin{gathered}
M=\left\{\xi \in \mathrm{T}: \int_{0}^{1}\left|f_{\widetilde{\tau}}(\tau \xi)\right|(1-\tau)^{\alpha} d \tau \geq \varepsilon\right\} . \\
M=M_{\varepsilon, \alpha, \widetilde{\tau}}(f)=M_{\varepsilon, \alpha} \subset \mathrm{T} ; \widetilde{\tau} \in(0,1), \alpha>0, \varepsilon>0 .
\end{gathered}
$$

Note first that from Bergman representation formula for large enough $\gamma$.

$$
\begin{aligned}
& \int_{0}^{1}|f(\tau \xi)|(1-\tau)^{\alpha} d \tau \\
\leq & C \int_{0}^{1} \int_{\mathbb{D}} \frac{|f(w)|(1-|w|)^{\gamma} d A(w)}{\left|(1-\tau \xi \bar{w})^{\gamma+2}\right|}(1-\tau)^{\alpha} d \tau \\
\leq & C_{1} \int_{\mathbb{D}} \frac{|f(w)|(1-|w|)^{\gamma} d A(w)}{|1-\xi \bar{w}|^{\gamma+1-\alpha}} ; \gamma>-1, \alpha>-1 .
\end{aligned}
$$

So we have

$$
\left\|\int_{0}^{1}|f(\tau \xi)|(1-\tau)^{\alpha} d \tau\right\|_{L^{\infty}(\mathrm{T})} \leq C \int_{\mathbb{D}}|f(w)|(1-|w|)^{\alpha-1} d A(w),
$$

where $f \in H(\mathbb{D}), \alpha>0$.

The problem of finding of estimates $\operatorname{dist}_{X}(f, Y), f \in A_{\alpha}^{\infty}$, where

$$
\begin{aligned}
& X=\left(A_{\alpha}^{\infty}\right)=\left\{f \in H(\mathbb{D}):\left\|\int_{0}^{1}|f(\tau \xi)|(1-\tau)^{\alpha} d \tau\right\|_{L^{\infty}}<\infty\right\}, \\
& Y=\left(A_{\alpha-1}^{1}\right)=\left\{f \in H(\mathbb{D}): \int_{0}^{1}|f(w)|(1-|w|)^{\alpha-1} d A(w)<\infty\right\}
\end{aligned}
$$

appears naturally.

Our intention is to find an analogue of a theorem Zhao for this situation, using characteristic function of $M$ set. 
By well-known Bergman representation formula (see [2]) for every $t>-1$

$$
\begin{aligned}
f(w)= & C(t) \int_{\mathbb{D}} \frac{f(z)(1-|z|)^{t} d A(z)}{(1-\bar{z} w)^{t+2}} \\
= & C(t) \int_{M_{\varepsilon, \alpha}(f)} \int_{0}^{1} \frac{f(\tau \xi)(1-\tau)^{t} d \xi d \tau}{(1-\tau \bar{\xi} \rho \varphi)^{t+2}} \\
& +C(t) \int_{0}^{1} \int_{T \backslash M_{\varepsilon, \alpha}(f)} \frac{f(\tau \xi)(1-\tau)^{t} d \xi d \tau}{(1-\tau \bar{\xi} \rho \varphi)^{t+2}} \\
= & f_{1}(w)+f_{2}(w) .
\end{aligned}
$$

We now estimate $\left(f_{1}\right)$ and $\left(f_{2}\right)$ separately.

Note first arguing as above we easily find that

$$
\begin{aligned}
\|f\|_{A_{\alpha, s}^{\infty}} & =\sup _{\tau<1}\left\|\int_{0}^{1}\left|f_{\tau}(\rho \xi)\right|(1-\rho)^{\alpha} d \rho\right\|_{L^{\infty}(\mathrm{T})}(1-\tau)^{s} \\
& \leq C \sup _{\tau<1}(1-\tau)^{s} \int_{\mathbb{D}}\left|f_{\tau}(w)\right|(1-|w|)^{\alpha-1} d A(w) \\
& =C\|f\|_{A_{\alpha-1, s}^{1}} .
\end{aligned}
$$

Hence the problem of estimate of $\operatorname{dist}_{A_{\alpha, s}^{\infty}}\left(f, A_{\alpha-1, s}^{1}\right), f \in A_{\alpha, s}^{\infty}$ also appears. Note for $s=0$, we have as before $\operatorname{dist}_{A_{\alpha, 0}^{\infty}}\left(f, A_{\alpha-1,0}^{1}\right)=\operatorname{dist}_{A_{\alpha}^{\infty}}\left(f, A_{\alpha-1}^{1}\right)$.

It turns out that for $s>0$ case, there is an opportunity to obtain a result of above mentioned type that is to give estimates for distance function. For that reason we will turn to estimates of $f_{1}$ and $f_{2}$ functions defined above. We will prove the two following inequalities, $s>0, \alpha>0$,

$$
\begin{aligned}
& \text { (A) }\left\|f_{2}\right\|_{A_{\alpha, s}^{\infty}} \leq C \varepsilon ; \\
& \text { (B) }\left\|f_{1}\right\|_{A_{\alpha-1, s}^{1}} \leq C_{1}\|f\|_{A_{\alpha, s}^{\infty} \sup _{\tau<1}(1-\tau)^{\widetilde{\tau}} \int_{\mathrm{T}} \chi_{M_{\varepsilon, t, \tau}}(\xi) d \xi} \\
& =C_{1}\|f\|_{A_{\alpha, s}^{\infty}} \cdot\left(K_{\alpha, t, \varepsilon}\right),
\end{aligned}
$$

where $\widetilde{\tau}=\alpha-t-1, K=\left(K_{\alpha, t, \varepsilon}\right)$.

We now will prove $(A)$ and then $(B)$. We have the following estimates.

$$
\begin{aligned}
& \left\|\int_{0}^{1}\left|f_{2}(\widetilde{\tau} \rho \varphi)\right|(1-\rho)^{\alpha} d \tau\right\|_{L^{\infty}(\mathrm{T})}(1-\widetilde{\tau})^{s} \\
\leq & C(1-\widetilde{\tau})^{s}\left\|\int_{0}^{1} \int_{\mathrm{T} \backslash M} \frac{|f(\widetilde{\tau} \xi)|(1-\tau)^{t} d \xi d \tau}{|1-\widetilde{\tau} \tau \bar{\xi} \varphi|^{t+1-\alpha}}\right\|_{L^{\infty}(\mathrm{T})} \\
\leq & C(1-\widetilde{\tau})^{s}\left\|\int_{\mathrm{T} \backslash M}\left(\int_{0}^{1}\left|f_{\widetilde{\tau}}(\tau \xi)\right|(1-\tau)^{t} d \tau\right) \frac{d \xi}{|1-\bar{\xi} \varphi \widetilde{\tau}|^{t+1-\alpha}}\right\|_{L^{\infty}(\mathrm{T})} \\
\leq & C \varepsilon .
\end{aligned}
$$

for $s=t-\alpha, s>0$ and $M=M_{\varepsilon, t, \tilde{\tau}}$ set that we defined above. 
Let us estimate the second integral. We have the following estimates.

$$
\begin{aligned}
\left\|f_{1}\right\|_{A_{\alpha-1, s}^{1}} \leq & C \sup _{\widetilde{\tau}<1}(1-\widetilde{\tau})^{s} \int_{\mathbb{D}}\left|f_{1}(w \widetilde{\tau})\right|(1-|w|)^{\alpha-1} d A(w) \\
\leq & C \sup _{\widetilde{\tau}<1}(1-\widetilde{\tau})^{s} \int_{M} \int_{0}^{1} \frac{|f(\tau \widetilde{\tau} \xi)|(1-\tau)^{t} d \xi d \tau}{(1-\widetilde{\tau})^{t-\alpha+1}} \\
\leq & C \sup _{\widetilde{\tau}<1}(1-\widetilde{\tau})^{s}\left\|\int_{0}^{1}|f(\tau \widetilde{\tau} \xi)|(1-\tau)^{t} d \tau\right\|_{L^{\infty}(\mathrm{T})} \\
& \cdot \sup _{\widetilde{\tau}<1} \int_{M} \frac{1}{(1-\widetilde{\tau})^{t-\alpha+1}} d \xi,
\end{aligned}
$$

where $s=t-\alpha, s>0, t>\alpha, M=\left(M_{\varepsilon, t, \tilde{\tau}}\right)$.

Hence

$$
\operatorname{dist}_{A_{\alpha, s}^{\infty}}\left(f, A_{\alpha-1, s}^{1}\right) \leq C \inf \{\varepsilon>0, K<\infty .\}
$$

Since

$$
\operatorname{dist}_{A_{\alpha, s}^{\infty}}\left(f, A_{\alpha-1, s}^{1}\right) \leq\left\|f-f_{1}\right\|_{A_{\alpha, s}^{\infty}}=\left\|f_{2}\right\|_{A_{\alpha, s}^{\infty}} \leq C \varepsilon .
$$

Hence we have the following theorem.

Theorem 2.2. Let $f \in A_{\alpha, s}^{\infty}(\mathbb{D}), \alpha>0, s=t-\alpha, t>\alpha, s>0$,

$$
\operatorname{dist}_{A_{\alpha, s}^{\infty}}\left(f, A_{\alpha-1, s}^{1}\right) \leq C \inf \left\{\varepsilon>0: \sup _{\widetilde{\tau}<1}(1-\widetilde{\tau})^{\alpha-t-1} \int_{\mathrm{T}} \chi_{M_{\varepsilon, t, \tilde{\tau}}}(\xi) d \xi<\infty .\right\}
$$

where

$$
M_{\varepsilon, t, \widetilde{\tau}}=\left\{\xi \in \mathrm{T}: \int_{0}^{1}\left|f_{\widetilde{\tau}}(\tau \xi)\right|(1-\tau)^{t} d \tau \geq \varepsilon\right\}
$$

Remark 2.3. Using arguments from [10](see also the proof of Theorem 2.4 below) it can be easily shown that for $s=0$ the assertion of Theorem 1 is sharp and that is the reverse estimate holds.

Let us now turn to another problem connected with distances. We will give sharp formula for dist function in BMOA-type classes. The following estimates are almost obvious.

Let $\beta>-1, \tau>0, \alpha>0, q>0$. Then we have

$$
\begin{aligned}
& \|f\|_{K}=\|f\|_{K(q, \alpha, \beta, \tau)} \\
\leq & \sup _{a \in \mathbb{D}} \int_{\mathbb{D}} \frac{|f(z)|^{q}(1-|z|)^{\beta} d A(z)}{|1-\bar{a} z|^{\alpha}}(1-|a|)^{\tau} \\
\leq & \left\{\begin{array}{l}
\|f\|_{K_{1}}=\sup _{|z|<1}|f(z)|^{q}(1-|z|)^{\beta}, \text { if } \alpha>2, \tau=\alpha-2 ; \\
\|f\|_{K_{2}}=\int_{\mathbb{D}}|f(z)|^{q}(1-|z|)^{\beta-\alpha+\tau} d A(z), \text { if } \alpha>\tau, \beta-\alpha+\tau>-1 .
\end{array}\right.
\end{aligned}
$$

The natural question is to estimate

$$
\operatorname{dist}_{K(q, \alpha, \beta, \tau)}\left(f, K_{1}\right) \text { and } \operatorname{dist}_{K(q, \alpha, \beta, \tau)}\left(f, K_{2}\right) \text { for }\|f\|_{K}<\infty .
$$

In the following assertion we provide such an estimate. 
First we define a new set, a subset of $\mathbb{D}$ as follows.

$$
N_{f, \varepsilon}=N_{q, \beta, \tau, \alpha, \varepsilon}(f)=\left\{a \in \mathbb{D}: \int_{\mathbb{D}} \frac{|f(z)|^{q}(1-|z|)^{\beta} d A(z)}{|1-\bar{a} z|^{\alpha}}(1-|a|)^{\tau} \geq \varepsilon\right\} .
$$

Put $f=\tilde{f}+f_{\varepsilon_{1}}$, assume $\|\widetilde{f}\|_{K}<\varepsilon$, then we find the following estimates.

$$
\begin{aligned}
M(a)= & \int_{\mathbb{D}} \frac{\left|f_{\varepsilon_{1}}(z)\right|^{q}(1-|z|)^{\beta} d A(z)}{|1-\bar{a} z|^{\alpha}}(1-|a|)^{\tau} \\
\geq & (1-|a|)^{\tau} \int_{\mathbb{D}} \frac{|f(z)|^{q}(1-|z|)^{\beta} d A(z)}{|1-\bar{a} z|^{\alpha}} \\
& \quad-\sup _{a \in \mathbb{D}} \int_{\mathbb{D}} \frac{|\widetilde{f}(z)|^{q}(1-|z|)^{\beta} d A(z)}{|1-\bar{a} z|^{\alpha}}(1-|a|)^{\tau} \\
\geq & (1-|a|)^{\tau} \int_{\mathbb{D}} \frac{|f(z)|^{q}(1-|z|)^{\beta} d A(z)}{|1-\bar{a} z|^{\alpha}}-\varepsilon_{1} .
\end{aligned}
$$

Hence $M(a) \geq\left(\varepsilon-\varepsilon_{1}\right) \chi_{N_{f, \varepsilon}}(a), a \in \mathbb{D}$.

Hence

$$
\begin{aligned}
& \int_{\mathbb{D}}\left|f_{\varepsilon_{1}}(z)\right|^{q}(1-|z|)^{\beta+\tau-\alpha} d A(z) \\
& \geq\left(\varepsilon-\varepsilon_{1}\right) \int_{\mathbb{D}} \chi_{N_{f, \varepsilon}}(a)(1-|a|)^{-2} d A(z),
\end{aligned}
$$

where $\tau>1, \beta+\tau-\alpha>-1, \alpha>\tau$.

Note from here we have immediately that

$$
\operatorname{dist}_{K(q, \alpha, \beta, \tau)}\left(f, K_{2}\right) \geq \inf \left\{\varepsilon: \int_{\mathbb{D}} \chi_{N_{f, \varepsilon}}(a)(1-|a|)^{-2} d A(z)<\infty, f \in K\right\} .
$$

Since otherwise there are two numbers $\varepsilon, \varepsilon_{1}, \varepsilon>0, \varepsilon_{1}>0$ such that

$$
f_{\varepsilon_{1}} \in K_{2}, \varepsilon>\varepsilon_{1},\left\|f-f_{\varepsilon_{1}}\right\|_{K(q, \alpha, \beta, \tau)} \leq \varepsilon_{1}
$$

and

$$
\int_{\mathbb{D}} \chi_{N_{f, \varepsilon}}(a)(1-|a|)^{-2} d A(z)=\infty
$$

and using estimates we provided above we arrive easily at a contradiction with the fact that

$$
\int_{\mathbb{D}} \chi_{N_{f, \varepsilon}}(a)(1-|a|)^{-2} d A(z)=\infty .
$$

Let $q=1, \beta=\alpha-2$. To prove the last estimate we obtained is sharp, we will argue as in Theorem 2.2 using classical Bergman representation formula. We cut the integral by $\mathbb{D}$ into two parts via $N_{f}$ sets and obtain two functions $f_{1}, f_{2}$ such that $f=f_{1}+f_{2}$. Then we will estimate both functions. Our intension is to prove the following estimates.

$$
\begin{aligned}
& \left\|f_{2}\right\|_{K} \leq C \varepsilon ; \quad(C) \\
& \left\|f_{1}\right\|_{K_{2}} \leq C\|f\|_{K} \cdot \int_{\mathbb{D}} \chi_{N_{f, \varepsilon}}(a)(1-|a|)^{-2} d A(z),
\end{aligned}
$$


$f=f_{1}+f_{2}$, where

$$
\begin{aligned}
& f_{1}\left(z_{1}\right)=C(\beta) \int_{\mathbb{D} \backslash N_{f, \varepsilon}} \frac{(1-|z|)^{\beta}}{\left(1-z_{1} \bar{z}\right)^{\beta+2}} \int_{\mathbb{D}} \frac{(1-|w|)^{\beta} f(w) d A(w)}{(1-\bar{w} z)^{\beta+2}} d A(z), \\
& f_{2}\left(z_{1}\right)=C(\beta) \int_{N_{f, \varepsilon}} \frac{(1-|z|)^{\beta}}{\left(1-z_{1} \bar{z}\right)^{\beta+2}} \int_{\mathbb{D}} \frac{(1-|w|)^{\beta} f(w) d A(w)}{(1-\bar{w} z)^{\beta+2}} d A(z) .
\end{aligned}
$$

$z_{1} \in \mathbb{D}, \beta>-1, f \in K$.

We will show first $(\mathrm{C})$, then $(\mathrm{D})$.

$$
\begin{aligned}
\left\|f_{2}\right\|_{K} \leq & \sup _{a \in \mathbb{D}} \int_{\mathbb{D}} \frac{\left(1-\left|z_{1}\right|\right)^{\beta}(1-|a|)^{\tau}}{\left|1-\bar{a} z_{1}\right|^{\alpha}} \\
& \cdot\left(\int_{\mathbb{D}} \int_{\mathbb{D} \backslash N_{f, \varepsilon}} \frac{|f(w)|(1-|z|)^{\beta}(1-|w|)^{\beta} d A(w)}{|1-z \bar{w}|^{\beta+2}\left|1-z z_{1}\right|^{\beta+2}} d A(z)\right) d A\left(z_{1}\right) .
\end{aligned}
$$

Then

$$
\begin{aligned}
\left\|f_{2}\right\|_{K} & \leq \varepsilon \sup _{a \in \mathbb{D}} \int_{\mathbb{D}} \frac{\left(1-\left|z_{1}\right|\right)^{\beta}(1-|a|)^{\tau}}{\left|1-\bar{a} z_{1}\right|^{\alpha}}\left(1-\left|z_{1}\right|\right)^{-\tau} d A\left(z_{1}\right) \\
& \leq \varepsilon \sup _{a \in \mathbb{D}} \int_{\mathbb{D}} \frac{|1-| z_{1}||^{\beta-\tau}(1-|a|)^{\tau}}{\left|1-\bar{a} z_{1}\right|^{\alpha}} d A\left(z_{1}\right) \\
& \leq C \varepsilon .
\end{aligned}
$$

Now we will prove (D).

$$
\begin{aligned}
\left|f_{1}\left(z_{1}\right)\right| \leq & C \int_{N_{f, \varepsilon}} \frac{(1-|z|)^{\beta}}{\left|1-z_{1} \bar{z}\right|^{\beta+2}}\left(\int_{\mathbb{D}} \frac{|f(w)|(1-|w|)^{\beta} d A(w)}{|1-\bar{w} z|^{\beta+2}}\right) d A(z) . \\
\left\|f_{1}\right\|_{K_{2}} \leq & C \int_{\mathbb{D}} \int_{\mathbb{D}} \frac{(1-|z|)^{\beta} \chi_{N_{f, \varepsilon}}(z)}{\left|1-z_{1} \bar{z}\right|^{\beta+2}} \\
& \cdot \int_{\mathbb{D}} \frac{|f(w)|(1-|w|)^{\beta} d A(w)}{|1-\bar{w} z|^{\alpha}} d A(z)\left(1-\left|z_{1}\right|\right)^{\beta-\alpha+\tau} d A\left(z_{1}\right) \\
\leq & C\|f\|_{K} \int_{\mathbb{D}} \int_{\mathbb{D}} \frac{\chi_{N_{f, \varepsilon}}(z)(1-|z|)^{\beta-\tau} d A(z)}{\left|1-z_{1} \bar{z}\right|^{\beta+2}}\left(1-\left|z_{1}\right|\right)^{\beta-\alpha+\tau} d A\left(z_{1}\right) \\
\leq & C\|f\|_{K} \int_{\mathbb{D}} \chi_{N_{f, \varepsilon}}(z)(1-|z|)^{-2} d A(z) .
\end{aligned}
$$

Since $\beta=\alpha-2$ and $\beta-\alpha+\tau=\tau-2, \tau>1$. This is what was needed. Hence we have Theorem 2.4 on dist function.

Theorem 2.4. Let $\alpha>1, \tau>1, \tau \in(\alpha-2, \alpha-1)$. Let

$$
\begin{aligned}
& \|f\|_{K(\alpha, \tau)}=\sup _{a \in \mathbb{D}} \int_{\mathbb{D}} \frac{|f(z)|(1-|z|)^{\alpha-2}}{|1-\bar{a} z|^{\alpha}} d A(z)(1-|a|)^{\tau} ; \\
& \|f\|_{A_{\tau-2}^{1}}=\int_{\mathbb{D}}|f(z)|(1-|z|)^{\tau-2} d A(z) .
\end{aligned}
$$

Let $\|f\|_{K(\alpha, \tau)}<\infty$. Then 


$$
\operatorname{dist}_{K}\left(f, A_{\tau-2}^{1}\right) \asymp \inf \left\{\varepsilon: \int_{\mathbb{D}} \chi_{N_{f, \varepsilon}}(a)(1-|a|)^{-2} d A(a)<\infty\right\} .
$$

Corollary 2.5. 1) Let $\alpha>1, \tau_{1}>1, \tau_{2}>1, \tau_{1} \in(\alpha-2, \alpha-1), \tau_{2} \in(\alpha-2, \alpha-$ $1), f \in K\left(\alpha, \min \left(\tau_{1}, \tau_{2}\right)\right)$. Then

$$
\operatorname{dist}_{A_{\tau_{1}-2}^{1}}\left(f, K\left(\alpha, \tau_{1}\right)\right)=\operatorname{dist}_{A_{\tau_{2}-2}^{1}}\left(f, K\left(\alpha, \tau_{2}\right)\right) .
$$

2) Let $\alpha_{1}>1, \alpha_{2}>1, \tau>1, \tau \in\left(\alpha_{1}-2, \alpha_{1}-1\right), \tau \in\left(\alpha_{2}-2, \alpha_{2}-1\right)$. Then

$$
\operatorname{dist}_{A_{\tau-2}^{1}}\left(f, K\left(\alpha_{1}, \tau\right)\right)=\operatorname{dist}_{A_{\tau-2}^{1}}\left(f, K\left(\alpha_{2}, \tau\right)\right)
$$

Corollary 2.6. Let $\tau \in(\alpha-2, \alpha-1), \alpha>1, \tau>1, f \in H(D)$. Then $f$ is in closure of $A_{\tau-2}^{1}$ in $K(\alpha, \tau)$ is equivalent to

$$
\int_{\mathbb{D}} \chi_{N_{f, \varepsilon}}(a)(1-|a|)^{-2} d A(a)<\infty
$$

for every $\varepsilon>0$.

Remark 2.7. Some results of this paper can be easily generalized to more general radial weights $w(1-|z|)$ with some natural conditions on $w$ function.

Acknowledgements: This work is partially supported by the the Key Project of Chinese Ministry of Education(No.208081) and the Natural Science Foundation of Henan(No. 2007110016; 2008B110006).

\section{REFERENCES}

1. L. V. Ahlfors, Bounded analytic functions, Duke Math Journal, 14( 1947), 1-14. 1

2. M. M. Djrbashian, F. A. Shamoian, Topics in theory of $A_{\alpha}^{p}$ classes, Teubner zur Mathematics, 1988, v105. 1, 2

3. P. L. Duren, Theory of $H^{p}$ spaces, Academic Press, New York, 1970. 1

4. P. G. Ghatage, D. Zheng, Analytic functions of bounded mean oscillation and the Bloch space, Integr. Equat. Oper. Theory, 17 (1993), 501-515. 1

5. D. Khavinson, M. Stessin, Certain linear extremal problems in Bergman spaces of analytic function, Indiana Univ. Math. J., 3(46) (1997), 933-974. 1

6. S. Ya Khavinson, On an extremal problem in the theory of analytic function, Russian Math. Surv., 4(1949), 158-159. 1

7. W. Rudin, Analytic functions of class $H^{p}$, Trans AMS, 78 (1955), 46-66. 1

8. W. Rudin, Functionn theory in polydisks, Benjamin, New York, 1969.

9. W. Xu, Distances from Bloch functions to some Möbius invariant function spaces in the unit ball of $C^{n}$, J. Funct. Spaces Appl., 7(1) (2009), 91-104. 1

10. R. Zhao, Distances from Bloch functions to some Möbius invariant function spaces, Ann. Acad. Sci. Fenn. Math., 33 (2008), 303-313. 1, 2.3

11. K. Zhu, Spaces of Holomorphic Functions in the Unit Ball, Graduate Texts in Mathematics, 226, Springer-Verlag, New York, 2005. 
1 Department of Mathematics, Bryansk State University, Bryansk 241050, RusSIA.

E-mail address: rsham@mail.ru

2 College of Mathematics and Information Science, Henan Normal University, Xinxiang 453007, P.R.China. ;

AMIRKABIR UNIVERSITY

E-mail address: tslhy2001@yahoo.com.cn 